\title{
Living Alone and Gender Differences in Rehospitalization for Heart Failure After Discharge Among Acute Heart Failure Patients
}

\author{
Kensuke Takabayashi, ${ }^{1}$ MD, Shouji Kitaguchi, ${ }^{1}$ MD, Kotaro Iwatsu, ${ }^{2}$ BSc, Tsutomu Ikeda, ${ }^{2}$ BSc, \\ Ryoko Fujita, ${ }^{1}$ MD, Miyuki Okuda, ${ }^{1} \mathrm{MD}$, Osamu Nakajima, ${ }^{3} \mathrm{MD}$, Hitoshi Koito, ${ }^{4} \mathrm{MD}$, \\ Yuka Terasaki, ${ }^{5} \mathrm{MD}$, Tetsuhisa Kitamura, ${ }^{6} \mathrm{MD}$ and Ryuji Nohara, ${ }^{1} \mathrm{MD}$
}

\begin{abstract}
Summary
Home treatment for heart failure (HF) is one of the most important problems in patients after discharge as a secondary preventive measure for rehospitalization for HF. However, there are no detailed studies on gender differences in sociopsychological factors such as living alone for HF rehospitalization among patients with acute HF (AHF).

This prospective multicenter cohort study enrolled patients with AHF between April 2015 and August 2017. Patients of each gender with first AHF were divided into those living alone and those not living alone. The primary endpoint was defined as rehospitalization for HF after discharge. Cox proportional hazard analysis was performed to determine the association between living alone and the endpoint.

Overall, 581 patients were included in this study during the 3-year follow-up. The proportion of rehospitalization for HF was significantly higher in patients living alone than in those not living alone among male patients. However, female patients showed no difference in endpoints between the two groups. The difference was independently maintained even after adjusting for differences in social backgrounds in male patients (adjusted hazard ratio (HR) 2.02; 95\% confidence interval (CI), 1.07-3.70). In female patients, the HR for rehospitalization for HF showed no difference between the two groups (adjusted HR, 0.99; 95\% CI, 0.56-1.69).

In this study population, male patients living alone after first AHF discharge had a higher risk of rehospitalization for HF than those not living alone, but these differences were not observed in female patients.
\end{abstract}

Key words: Social factor, Living style, Male, Female, Sex, Prognosis, Cohort study

(Int Heart J 2020; 61: 1245-1252)

$\mathrm{I}$ n 2018, Japan had the highest proportion of elderly people in the world. ${ }^{1,2)}$ Home treatment for heart failure (HF) is one of the most important problems in elderly patients after discharge as a secondary preventive measure for rehospitalization for $\mathrm{HF}^{3)}$ However, the impact of sociopsychological factors on rehospitalization for HF or mortality after discharge in patients with acute HF (AHF) has not been well investigated. Several studies have investigated the role of sociopsychological factors and outcomes in cardiovascular diseases. Living alone was reported to be an independent predictor of mortality in patients with $\mathrm{HF}^{4}{ }^{4}$ and unmarried patients with $\mathrm{HF}$ were found to have significantly higher 30-day readmission and 1-year mortality rates than married patients. ${ }^{5)}$ Contrarily, a Japanese study demonstrated that, during 1-year followup, living alone was not an independent predictor of readmission in patients with chronic congestive HF. ${ }^{6}$ The HF-
ACTION study showed that having a partner was not independent predictors of long-term clinical outcomes in patients with chronic HF. ${ }^{7}$ Some studies have mentioned that living alone was associated with a higher risk of cardiovascular events after discharge among patients with acute myocardial infarction (AMI) during the long-term follow-up in Japan and Western countries. ${ }^{89}$ In several parts of the world, family members undertake the majority of tasks of caring for elderly people in the community. ${ }^{10,11)}$ It has also been reported that significant differences in responsibility for household tasks between genders in the traditional Japanese household exist. ${ }^{10)}$ Despite the increase in the number of elderly people living alone in Japan with the rapid aging of society, no prospective studies have explained the relationship between living alone and the long-term prognosis of patients with AHF in Japan. Therefore, this study was conducted to determine whether

From the ${ }^{1}$ Department of Cardiology, Hirakata Kohsai Hospital, Osaka, Japan, ${ }^{2}$ Department of Rehabilitation, Hirakata Kohsai Hospital, Osaka, Japan, ${ }^{3}$ Department of Cardiology, Hirakata City Hospital, Osaka, Japan, ${ }^{4}$ Department of Cardiology, Otokoyama Hospital, Kyoto, Japan, ${ }^{5}$ Department of Internal Medicine, Arisawa General Hospital, Osaka, Japan and ${ }^{6}$ Department of Social and Environmental Medicine, Graduate School of Medicine, Osaka University, Osaka, Japan.

This work was supported by research funding from Nakajima Steel Pipe Company Limited.

Address for correspondence: Kensuke Takabayashi, MD, Department of Cardiology, Hirakata Kohsai Hospital, 1-2-1, Fujisakahigashimachi, Hirakata, Osaka 573-0153, Japan. E-mail: taka410@gmail.com

Received for publication June 3, 2020. Revised and accepted July 16, 2020

Released in advance online on J-STAGE November 13, 2020.

doi: $10.1536 /$ ihj. $20-386$

All rights reserved by the International Heart Journal Association. 


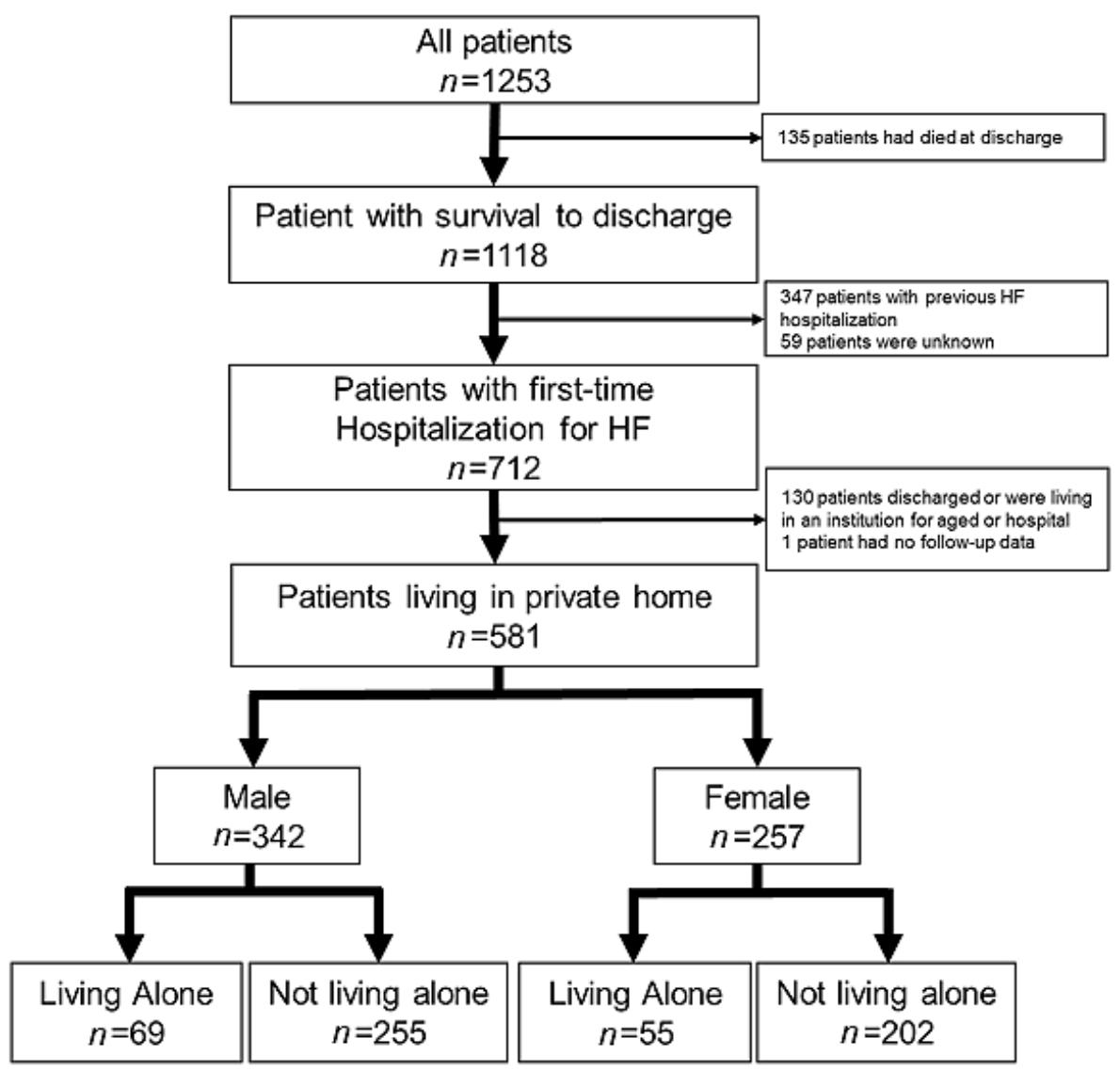

Figure 1. The study flow diagram. We enrolled 1253 patients with acute heart failure, of which 671 were excluded; finally, among the 582 patients who were discharged to their home, 581 had available follow-up data. We had 69 male patients living alone, 255 male patients not living alone, 55 female patients living alone, and 202 patients not living alone.

living alone could be an independent prognostic risk factor for long-term outcomes and gender differences in the risk for the endpoints in patients with first AHF.

\section{Methods}

We analyzed the data of patients with AHF from the Kitakawachi Clinical Background and Outcome of Heart Failure (KICKOFF) Registry, a prospective, multicenter, community-based cohort of Japanese patients with acute $\mathrm{HF}{ }^{12,13)}$ which registered patients hospitalized from April 2015 to August 2017. Overall, 13 hospitals, which consisted of 1 cardiovascular center and 12 small- or medium-sized hospitals, and were located north of Kitakawachi, at the eastern end of Osaka Prefecture, and Yawata, at the southern end of Kyoto Prefecture, participated in the Registry. During the study period, the northern parts of Kitakawachi and Yawata had a total population of 798,000 and were typical satellite communities in Japan. Patients were diagnosed with HF based on the Framingham criteria, i.e., the presence of at least two of the major criteria or one major and two minor criteria. ${ }^{14)}$ There were no exclusion criteria. The detailed study design of the KICKOFF Registry is presented in the UMIN Clinical Trials Registry (UMIN000016850). The clinical data of all patients were uploaded to the Electronic Data Capture system and automatically checked for missing or contradictory entries and values not in the normal range by the physicians in charge at each institution. The general office of the Registry checked all the collected data after registration. Data obtained from medical record reviews and interviews with patients or other family members were collected.

Overall, 1253 patients with AHF were enrolled in this Registry. In the present study, among the 1118 patients who were discharged, 347 were excluded because they had been hospitalized for HF before the registration and because patients with first HF hospitalization might not have less chance to prepare their sufficient living environment, for example, family support or social services, until discharge than those with previous HF hospitalization, and 59 were excluded because they lacked detailed information of hospitalization for HF. Of the remaining 712 patients, 130 who were hospitalized or institutionalized were excluded. Finally, among the 582 patients who were discharged to their home, 581 had available followup data (Figure 1). Based on the living status at discharge, patients were divided into two groups: patients who lived with their family or others (not living alone) and those who did not live with their family or others (living alone). 
Definitions of comorbidities have been described in our previous report. ${ }^{12}$ The primary dietary manager was defined as the person who most frequently provided the patient with his/her meals, which can be the patient's own or others. The primary drug therapy manager was defined as the person who most frequently managed the patient's drug therapy on a daily basis, which can be the patient's own or others. In this study, we defined the eligibility of long-term care insurance (LTCI) as patients who had LTCI certification, including both all support level and all care level, at discharge.

Follow-up data of outcomes were obtained at 6 and 12 months after discharge of AHF and annually thereafter for 3 years. Follow-up data were prospectively collected primarily by a review of hospital records, and additional follow-up information was obtained via telephone or mail contact with the patients or their relatives.

The primary outcome of this study was rehospitalization for HF during the follow-up period. Other clinical outcomes were defined as all-cause mortality and a composite endpoint consisting of rehospitalization for $\mathrm{HF}$ and all-cause mortality. ${ }^{15)}$

The investigation conforms with the principles outlined in the Declaration of Helsinki and was approved by the ethics committee of the Hirakata Kohsai Hospital (Osaka, Japan). Informed consent was obtained from all patients for their enrollment in the Registry. To preserve patient confidentiality, direct patient identifiers were not registered. The study did not alter any treatment specified in the protocol or any other method of outpatient care.

In this study population, the baseline clinical characteristics were compared between patients with first AHF living alone at their home and those not living alone according to gender. We also divided the study population into subgroups of elderly (aged $\geq 65$ years) and nonelderly (aged $<65$ years) patients according to previous studies. ${ }^{8,16)}$ Continuous variables are expressed as mean \pm standard deviation or as median with $25 \%-75 \%$ quartile and were compared using Student's $t$-test. Categorical variables are expressed as numbers and percentages and were compared using a chi-squared test. The Kaplan-Meier method was used to evaluate the cumulative incidences of hospitalization for HF, all-cause mortality, and the composite endpoint. The estimated differences were compared by a log-rank test. In addition, multivariable analysis, using a Cox proportional hazard model, was performed to evaluate the association between living alone and the incidence of outcomes. The timing of each event from discharge was identified. If there were no events in each patient in the 3 years of follow-up as of March 2019, censoring was conducted. Hazard ratios (HRs) and their 95\% confidence interval (CI) were also calculated. To evaluate the effects of living alone, two multivariable models considered to be associated with the clinical outcomes were adjusted as follows: model 1 was adjusted for age (/10 years for all patients) and comorbidities (yes or no), such as history of coronary artery disease, hypertension, diabetes mellitus, dyslipidemia, atrial fibrillation, chronic kidney disease, and stroke, and model 2 was adjusted for age (/10 years for all patients) and lifestyle factors (yes or no), such as current smoker, daily drinking, primary dietary manager (own), primary drug therapy manager (own), and eligibility of LTCI. Based on previous studies, those factors were included in the multivariable models. ${ }^{4-6,16)}$ All statistical analyses were performed using JMP version 14 (SAS Institute, Cary, NC, USA). All tests were two-tailed, and $P$ values of $<0.05$ were considered as statistically significant.

\section{Results}

The baseline clinical characteristics of patients living alone and those not living alone according to gender are shown in Table I. In terms of male gender $(n=342)$, no significant difference was observed in mean age or the proportion of comorbidities between the groups. Regarding females $(n=257)$, patients living alone were older in age and appeared to have less incidence of coronary artery disease, diabetes mellitus, dyslipidemia, or stroke than those not living alone. In both gender groups, the proportions of primary dietary managers of patients themselves and eligibility of LTCI were significantly higher in patients living alone than in those not living alone.

As of March 2019, the cumulative event rates were comparable between patients living alone and those not living alone. Among all patients, 149 (25.7\%) were hospitalized for HF again, and there was no significant difference between the proportion of rehospitalization for HF in patients living alone and those not living alone $(31.5 \%$ versus $24.1 \%, P=0.100)$. The results of the KaplanMeier analysis showed that the incidence of rehospitalization for HF was significantly higher in male patients living alone than in those not living alone, but there were no significant differences in the rates of other endpoints, allcause mortality, or the composite endpoint between the groups (Figure 2). Among female patients, there were no significant differences in the rate of each event. Regarding elderly patients, no significant differences were also noted in the incidence of each event among elderly female patients; however, there was a significantly higher incidence of rehospitalization for $\mathrm{HF}$ in patients living alone than in those not living alone among elderly male patients (Figure 3 ). In the nonelderly patients, no significant differences were observed in the endpoints.

In the Cox proportional hazard model (Table II), no significant difference was found in female patients in each model; however, in all the male patients, those living alone had a higher risk of rehospitalization for HF in each model (adjusted HR, 1.75; 95\% CI, 1.01-2.95, $P=0.036$ in model 1; adjusted HR, 2.02; 95\% CI, 1.07-3.70, $P=$ 0.032 in model 2). In elderly male patients, the HR of rehospitalization for HF also appeared to increase in each model (adjusted HR, 1.72; 95\% CI, 0.93-3.05, $P=0.085$; adjusted HR, 2.00; 95\% CI, 0.99-3.87, $P=0.053$ in model 2).

\section{Discussion}

This community-based prospective Registry study of patients with AHF in Japan demonstrated that male patients living alone had a significantly higher risk of rehospitalization for $\mathrm{HF}$ after discharge of first AHF than those 
Table I. Baseline Clinical Backgrounds

\begin{tabular}{|c|c|c|c|c|c|c|}
\hline & \multicolumn{3}{|c|}{ Male } & \multicolumn{3}{|c|}{ Female } \\
\hline & Living alone & Not living alone & & Living alone & Not living alone & \\
\hline$n$ & 69 & 255 & $P$ value & 55 & 202 & $P$ value \\
\hline Age (years) & $71.9 \pm 12.4$ & $72.3 \pm 11.2$ & 0.792 & $80.8 \pm 10.0$ & $77.2 \pm 12.1$ & 0.041 \\
\hline$\geq 65$ years & $49(71.0 \%)$ & $198(77.6 \%)$ & 0.259 & $52(94.5 \%)$ & $177(87.6 \%)$ & 0.117 \\
\hline BMI $\left(\mathrm{kg} / \mathrm{m}^{2}\right)$ & $22.5 \pm 3.9$ & $22.4 \pm 3.3$ & 0.931 & $21.2 \pm 3.8$ & $22.3 \pm 4.6$ & 0.115 \\
\hline \multicolumn{7}{|l|}{ Comorbidities } \\
\hline History of coronary artery disease & $20(29.0 \%)$ & $85(33.3 \%)$ & 0.491 & $7(12.7 \%)$ & $49(24.3 \%)$ & 0.054 \\
\hline Hypertension & $45(65.2 \%)$ & $184(72.2 \%)$ & 0.267 & $35(63.6 \%)$ & $143(70.8 \%)$ & 0.313 \\
\hline Diabetes mellitus & $27(39.1 \%)$ & $88(34.5 \%)$ & 0.479 & $11(20.0 \%)$ & $67(33.2 \%)$ & 0.052 \\
\hline Dyslipidemia & $31(44.9 \%)$ & $97(38.0 \%)$ & 0.302 & $16(29.1 \%)$ & $88(43.6 \%)$ & 0.049 \\
\hline Atrial fibrillation & $25(36.2 \%)$ & $86(33.7 \%)$ & 0.698 & $24(43.6 \%)$ & $79(39.1 \%)$ & 0.545 \\
\hline Chronic kidney disease & $34(49.3 \%)$ & $115(45.1 \%)$ & 0.537 & $25(45.5 \%)$ & $81(40.1 \%)$ & 0.476 \\
\hline History of stroke & $7(10.1 \%)$ & $22(8.6 \%)$ & 0.699 & $2(3.6 \%)$ & $21(10.4 \%)$ & 0.088 \\
\hline $\mathrm{BNP}(\mathrm{pg} / \mathrm{dL})$ & $\begin{array}{c}184.2 \\
{[77.4-319.0]}\end{array}$ & $\begin{array}{c}159.5 \\
{[68.4-377.1]}\end{array}$ & 0.882 & $\begin{array}{c}285.4 \\
{[82.7-535.3]}\end{array}$ & $\begin{array}{c}186.4 \\
{[88.8-458.2]}\end{array}$ & 0.621 \\
\hline $\operatorname{LVEF}(\%)$ & $49.0 \pm 16.9$ & $50.7 \pm 16.7$ & 0.456 & $58.8 \pm 14.3$ & $55.6 \pm 16.9$ & 0.206 \\
\hline LVEF $<50 \%$ & $33(50.0 \%)$ & $140(56.2 \%)$ & 0.367 & $39(76.5 \%)$ & $129(65.2 \%)$ & 0.116 \\
\hline Current smoker & $23(33.3 \%)$ & $61(23.9 \%)$ & 0.121 & $4(7.3 \%)$ & $12(5.9 \%)$ & 0.722 \\
\hline Daily drinking & $25(36.2 \%)$ & $77(30.2 \%)$ & 0.343 & $2(3.6 \%)$ & $12(5.9 \%)$ & 0.485 \\
\hline Main dietary manager (own) & $38(55.1 \%)$ & $32(12.6 \%)$ & $<0.001$ & $44(80.0 \%)$ & $116(57.4 \%)$ & 0.002 \\
\hline Main drug therapy manager (own) & $64(92.8 \%)$ & $222(87.1 \%)$ & 0.171 & $47(85.5 \%)$ & $159(78.7 \%)$ & 0.253 \\
\hline Family support & $24(34.8 \%)$ & N/A & & $35(63.6 \%)$ & N/A & \\
\hline Long-term care insurance & $15(21.7 \%)$ & $31(12.2 \%)$ & 0.053 & $27(49.1 \%)$ & $69(34.2 \%)$ & 0.045 \\
\hline
\end{tabular}

Categorical data are presented as number $(\%)$. Continuous variables are expressed as mean \pm standard deviation or as median [25\%-75\% quartile]. BMI indicates body mass index; BNP, brain natriuretic peptide; LVEF, left ventricle ejection fraction; and N/A, not applicable.

not living alone, but there was no significant difference among the female patients. Importantly, these differences were independently maintained even after adjusting for differences in comorbidities or social backgrounds. This study discloses the association between living alone and long-term outcomes in patients with AHF and added important information to the secondary prevention approach after AHF in Japan.

We observed that living alone was significantly associated with a higher risk of rehospitalization for HF following 3 years after discharge for AHF. Previous studies have reported that patients with HF living without a partner or those who were unmarried were at an increased risk for readmission at 90 days. ${ }^{17)}$ For the short-term outcome, the factor of marital status had a positive cumulative effect on the risk for readmission, ${ }^{18)}$ but the HFACTION study, which enrolled patients with chronic HF between 2003 and 2007 in the Western country, retrospectively showed that having a partner was not independent predictors of long-term clinical outcomes. ${ }^{7)}$ The patients in our study were older than those in the HF-ACTION study, and we focused on the association between outcomes and living alone, not partner status. Partner status was not same situation for living alone. The significances of social status or situation might differ based on culture, countries, and over time. During a long-term follow-up in Japan ${ }^{8)}$ and Western countries, ${ }^{9}$ other studies have demonstrated that living alone was significantly associated with adverse events following discharge in patients with AMI. These results are consistent with those of our study regarding the long-term outcome in patients with AHF. As a predictor of readmission in elderly patients with HF, social network, which was defined using a four-item questionnaire that includes living alone, was reported in Spain. ${ }^{19)}$ Patients not living alone provided a higher social network, and a higher social network was associated with a lower risk for readmission. Hence, focusing on sociopsychological components as an important factor for the secondary prevention of cardiovascular diseases is important.

In the present study, the higher rate of rehospitalization for HF after discharge in patients with first AHF who lived alone was observed only among male patients, whereas such a finding was not observed among female patients. Consistently, a previous study reported that, especially for the male gender and not the female gender, after adjusting for other risk factors, AMI survivors living alone had a significantly higher risk than those not living alone. ${ }^{8)}$ In the present study, male patients living alone had limited primary dietary management themselves than female patients $(55.1 \%$ in males and $80.0 \%$ in females living alone). Studies have shown that poor nutritional status and lack of sodium control were associated with an increased risk of adverse events among patients with $\mathrm{HF}^{20,21)}$ In addition, people living alone had poor dietary quality and patterns. ${ }^{22,23)}$ Female patients might manage well themselves after discharge for $\mathrm{HF}$ because they would be aware of the fact that one of the most important treatments was the control of daily diet during their admissions. We also observed that male patients living alone had less other family support or eligibility for LTCI than female patients $(34.8 \%$ in males and $63.6 \%$ in females or $21.7 \%$ in males and $49.1 \%$ in females, respectively). Family and social supports are effective factors in the management for preventing readmission for HF. Countries 
A

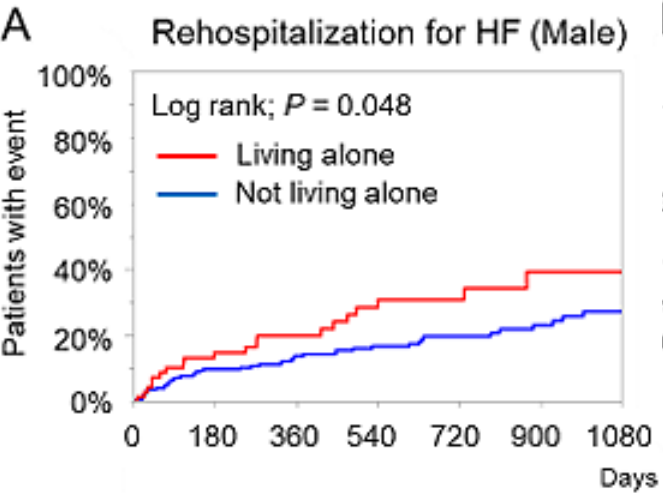

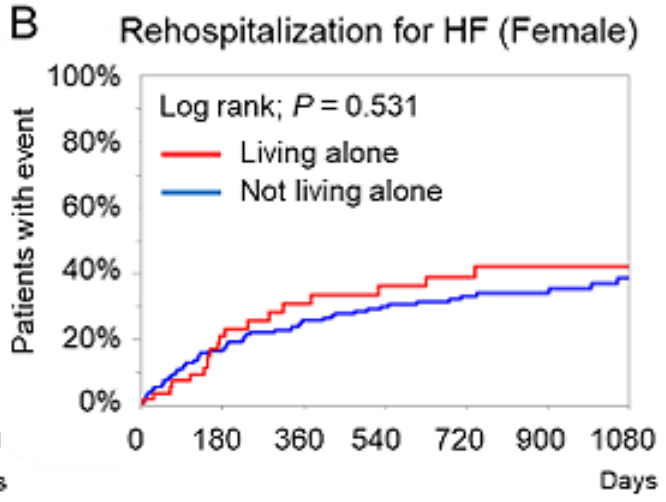
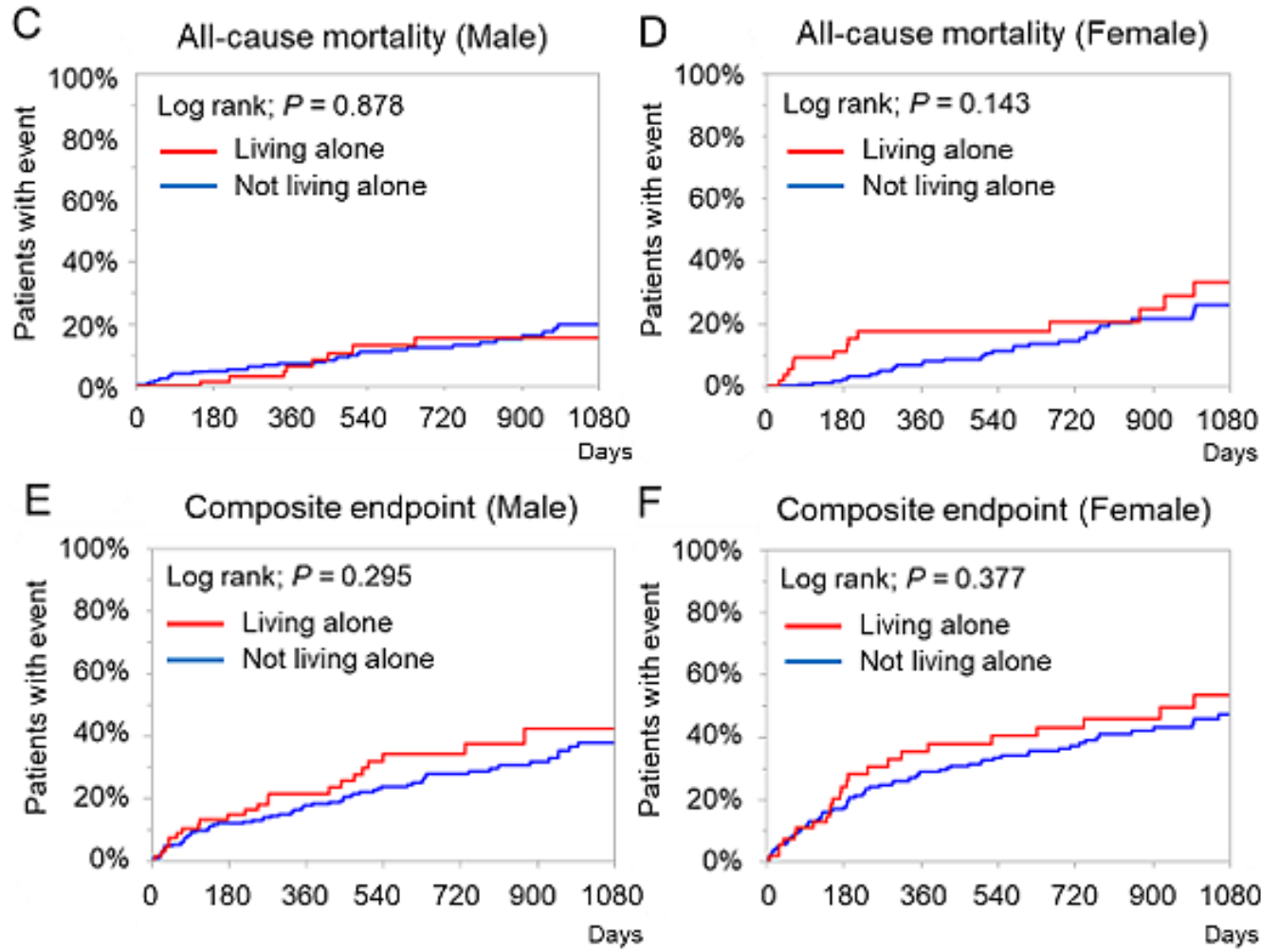

$\mathrm{F}$

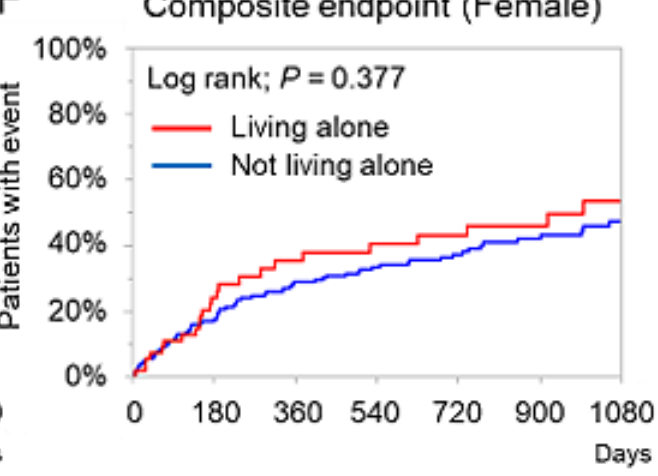

Figure 2. Kaplan-Meier curves for rehospitalization for HF, all-cause mortality, and the composite endpoint during the follow-up period between patients living alone and those not living alone among all patients. The rate of rehospitalization for heart failure was significantly higher in male patients living alone than in those not living alone (A), but there was no significant difference in the rate of other outcomes, all-cause mortality $(\mathbf{C})$, or the composite endpoint $(\mathbf{E})$ between the groups. In female patients, no significant difference was noted in the rate of each event $(\mathbf{B}, \mathbf{D}$, and $\mathbf{B})$.

throughout the world are facing the challenge of an aging population that will require care. ${ }^{24)}$ In these countries, including Japan, family members undertake the majority of caring tasks for elderly people in the community. ${ }^{25)}$ In Japan, the LTCI system helps people who require long-term care as a result of old age-induced illness, enabling them to lead more independent lives and relieve the burden of family care. ${ }^{10)}$ However, we could not find the effect of the just eligible of LTCI on the prevention of outcomes among patients with living alone because of our small samples. We would try to reveal the association between the LTCI and prognosis in a further study. Our results suggested that female patients could have more opportuni- ties to manage their daily living with some support from other younger family members or social support than male patients. Considering our study findings, we can recommend that patients with AHF use more family support or those services after discharge. In addition, social isolation tends to be associated with higher risk behaviors such as smoking and alcohol abuse. ${ }^{26)}$ In our study, although no significant differences in the proportion of current smokers and daily drinking history between patients living alone and those not living alone were found, the proportion of these risk behaviors was higher in males than in females. A cardiac rehabilitation program after discharge has potential to induce a better lifestyle and outcomes 
A
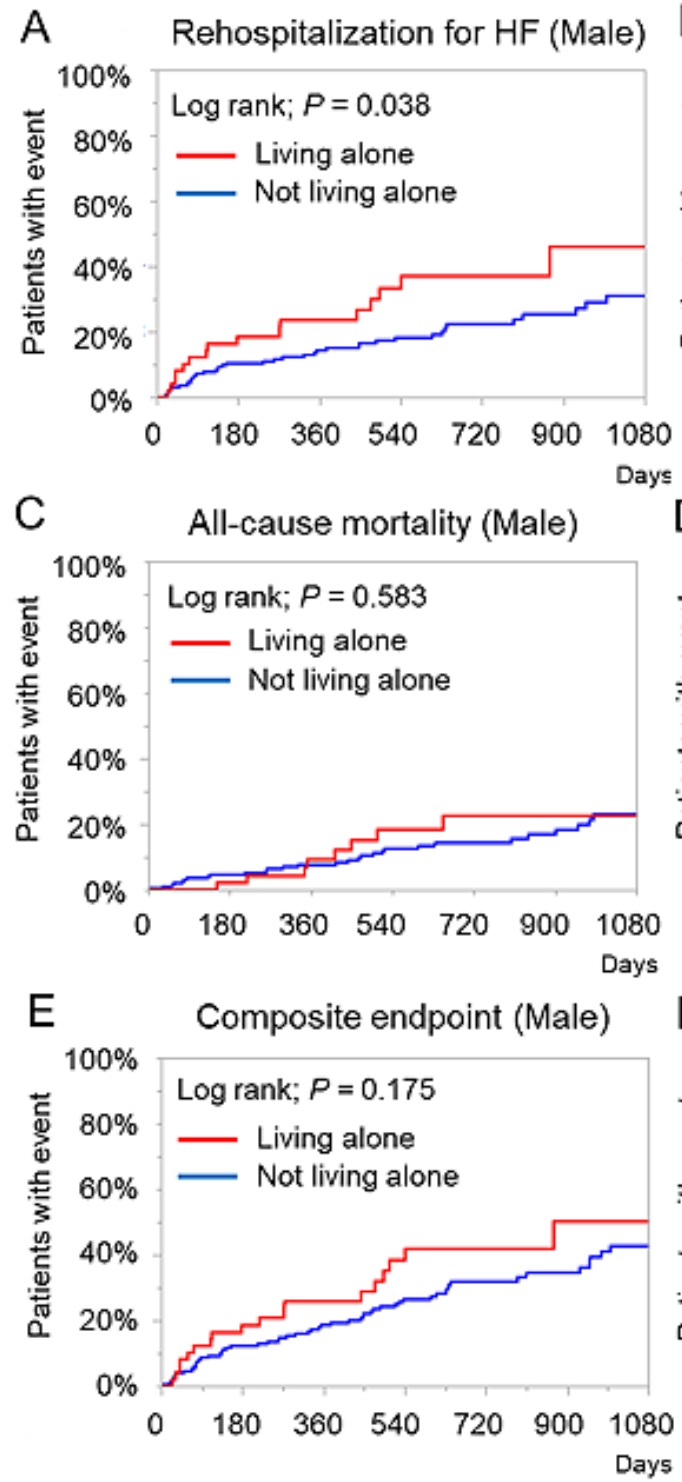
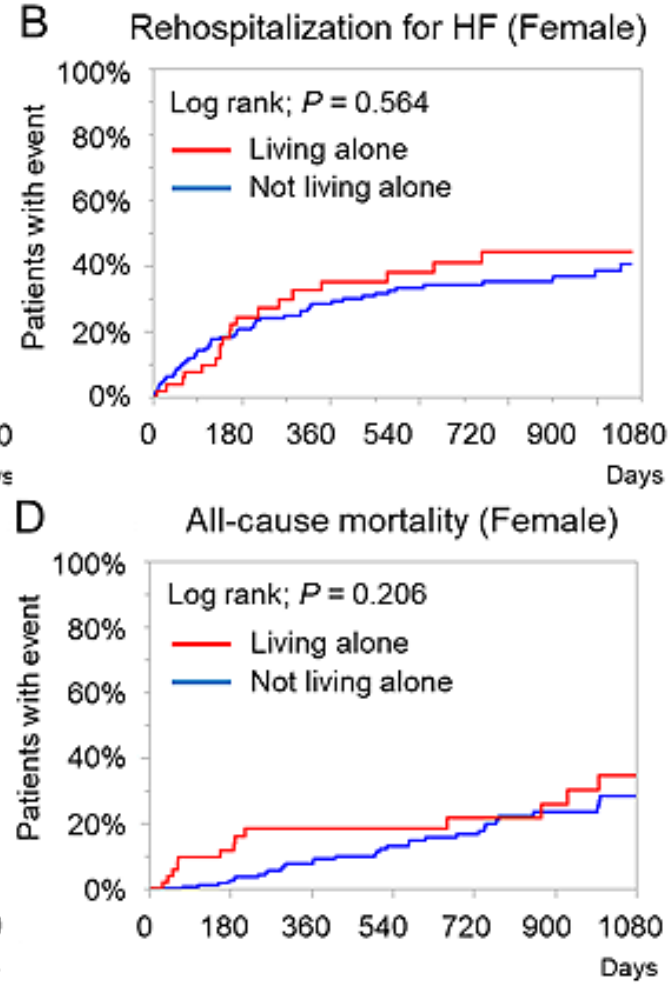

$\mathrm{F}$

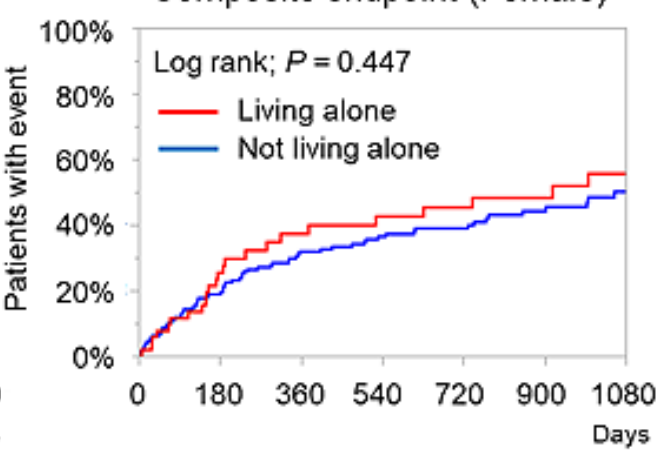

Figure 3. Kaplan-Meier curves for rehospitalization for heart failure, all-cause mortality, and the composite endpoint during the follow-up period between patients living alone and those not living alone among elderly patients. Regarding elderly patients, no significant difference was also noted in the rate of each event in elderly female patients $(\mathbf{B}, \mathbf{D}$, and $\mathbf{F})$; however, significantly higher rates of rehospitalization for heart failure were recorded in patients living alone than in those not living alone among elderly male patients (A).

among patients living alone. Unfortunately, we did not have data on the implementation of cardiac rehabilitation program during hospital stay and after discharge; however, it had been clear that the cardiac rehabilitation program in the acute phase of hospital stay and for outpatients with AHF to continue the program were effective on not only physical capacity but also quality of life or changing their lifestyle because of a comprehensive medical care. ${ }^{27}$ Several factors were associated with readmission in patients with AHF; however, noting that not living alone independently resulted in better outcomes after adjustments for comorbidities or social backgrounds, especially in male patients, is important.

We could not obtain the differences in all-cause death and the composite endpoint between living alone and not living alone. Most of the patients in this study were old, and they were older than those reported in previous Japanese registries. ${ }^{28,29)}$ Elderly patients with HF are more often exposed to the effects of various comorbidities and other risks inherent with aging. HF does not always immediately lead to death. HF repeats exacerbations and remissions. ${ }^{30)}$ In this study, the proportion of cardiac death was $36.8 \%$ and those of noncardiac death, excluding cancer death, was $39.1 \%$. All-cause death might be caused from many comorbidities or other diseases, especially in elderly patients, regardless of the living situation or support of others. No differences in the composite endpoint between living alone and not living alone might be af- 
Table II. Adjusted Hazard Ratios of Living Alone for Each Event during Follow-Up Period

\begin{tabular}{|c|c|c|c|c|c|c|c|c|c|c|}
\hline & \multicolumn{5}{|c|}{ Male } & \multicolumn{5}{|c|}{ Female } \\
\hline & \multirow[b]{2}{*}{$\begin{array}{c}\text { Event } \\
(n)\end{array}$} & \multicolumn{2}{|c|}{ Model 1} & \multicolumn{2}{|c|}{ Model 2} & \multirow[b]{2}{*}{$\begin{array}{c}\text { Event } \\
(n)\end{array}$} & \multicolumn{2}{|c|}{ Model 1} & \multicolumn{2}{|c|}{ Model 2} \\
\hline & & $\begin{array}{c}\text { HRs } \\
(95 \% \mathrm{CI})\end{array}$ & $\begin{array}{c}P \\
\text { value }\end{array}$ & $\begin{array}{c}\text { HRs } \\
(95 \% \mathrm{CI})\end{array}$ & $\begin{array}{c}P \\
\text { value }\end{array}$ & & $\begin{array}{c}\text { HRs } \\
(95 \% \mathrm{CI})\end{array}$ & $\begin{array}{c}P \\
\text { value }\end{array}$ & $\begin{array}{c}\text { HRs } \\
(95 \% \mathrm{CI})\end{array}$ & $\begin{array}{c}P \\
\text { value }\end{array}$ \\
\hline \multicolumn{11}{|c|}{ Rehospitalization for HF } \\
\hline All & 20 & $\begin{array}{c}1.75 \\
(1.01-2.95)\end{array}$ & 0.036 & $\begin{array}{c}2.02 \\
(1.07-3.70)\end{array}$ & 0.032 & 19 & $\begin{array}{c}0.91 \\
(0.52-1.53)\end{array}$ & 0.737 & $\begin{array}{c}0.99 \\
(0.56-1.69)\end{array}$ & 0.977 \\
\hline$\geq 65$ years & 16 & $\begin{array}{c}1.72 \\
(0.93-3.05)\end{array}$ & 0.085 & $\begin{array}{c}2.00 \\
(0.99-3.87)\end{array}$ & 0.053 & 19 & $\begin{array}{c}1.09 \\
(0.62-1.84)\end{array}$ & 0.760 & $\begin{array}{c}1.18 \\
(0.67-1.99)\end{array}$ & 0.551 \\
\hline$<65$ years & 4 & $\begin{array}{c}2.79 \\
(0.64-12.2)\end{array}$ & 0.165 & $\begin{array}{c}2.04 \\
(0.45-7.71)\end{array}$ & 0.332 & 0 & Unconverged & & Unconverged & \\
\hline \multicolumn{11}{|c|}{ All-cause death } \\
\hline All & 8 & $\begin{array}{c}0.98 \\
(0.41-2.07)\end{array}$ & 0.982 & $\begin{array}{c}1.19 \\
(0.47-2.69)\end{array}$ & 0.703 & 13 & $\begin{array}{c}1.35 \\
(0.65-2.68)\end{array}$ & 0.405 & $\begin{array}{c}1.54 \\
(0.74-3.04)\end{array}$ & 0.238 \\
\hline$\geq 65$ years & 8 & $\begin{array}{c}1.18 \\
(0.50-2.51)\end{array}$ & 0.675 & $\begin{array}{c}1.51 \\
(0.58-3.55)\end{array}$ & 0.379 & 13 & $\begin{array}{c}1.73 \\
(0.84-3.42)\end{array}$ & 0.135 & $\begin{array}{c}1.78 \\
(0.87-3.46)\end{array}$ & 0.109 \\
\hline$<65$ years & 0 & Unconverged & & Unconverged & & 0 & Unconverged & & Unconverged & \\
\hline \multicolumn{11}{|c|}{ Composite endpoint } \\
\hline All & 22 & $\begin{array}{c}1.37 \\
(0.82-2.20)\end{array}$ & 0.223 & $\begin{array}{c}1.63 \\
(0.92-2.79)\end{array}$ & 0.094 & 24 & $\begin{array}{c}0.94 \\
(0.57-1.51)\end{array}$ & 0.807 & $\begin{array}{c}1.02 \\
(0.61-1.64)\end{array}$ & 0.952 \\
\hline$\geq 65$ years & 18 & $\begin{array}{c}1.34 \\
(0.76-2.25)\end{array}$ & 0.305 & $\begin{array}{c}1.70 \\
(0.91-3.07)\end{array}$ & 0.095 & 24 & $\begin{array}{c}1.18 \\
(0.71-1.89)\end{array}$ & 0.515 & $\begin{array}{c}1.24 \\
(0.75-2.00)\end{array}$ & 0.379 \\
\hline$<65$ years & 4 & $\begin{array}{c}1.58 \\
(0.41-5.29)\end{array}$ & 0.481 & $\begin{array}{c}1.56 \\
(0.38-5.19)\end{array}$ & 0.505 & 0 & Unconverged & & Unconverged & \\
\hline
\end{tabular}

Event number was described in living alone patients. HRs of model 1 was adjusted for age (10-year strata for all patients) and comorbidities (yes/ no; coronary artery disease, hypertension, dyslipidemia, diabetes mellitus, atrial fibrillation, chronic kidney disease, stroke). HRs of model 2 was adjusted for age (10-year strata for all patients) and lifestyle factors (yes/no; current smoking, daily drinking, main dietary manager (own), main drug therapy manager (own), and eligibility of long-term care insurance). The composite endpoint included all-cause mortality and rehospitalization for HF. HF indicates heart failure; HRs, hazard ratios; and CI, confidence interval.

fected strongly from all-cause death event.

This Registry study has several limitations. First, there were no data on the changes in living arrangements after discharge during the follow-up period. Second, the diagnosis of AHF was defined by physicians using the Framingham criteria, which could consequently lead to possible selection or referral bias. Third, patients who had a history of HF before admission were excluded; however, we believed that patients with AHF with first admission had been more affected on living arrangement or support systems than those with readmission. Fourth, no event of all-cause death in male patients aged $<65$ years and in female patients aged $<65$ years was recorded in this study. We could not determine the presence of an association with outcome in those subgroups. Therefore, we anticipate that future studies would reveal the association to assess more clearly the association of outcomes with living alone, especially in patients aged $<65$ years.

In conclusion, this study that was conducted in Japan demonstrated that male patients living alone after discharge for AHF had a higher risk of rehospitalization for $\mathrm{HF}$ than those not living alone, but these differences were not observed in female patients. Focusing on the living style of the patient as an important factor for the secondary prevention of rehospitalization for $\mathrm{HF}$ and considering using family or social support, especially in male patients with first HF, are important.

\section{Acknowledgment}

We sincerely appreciate the help of all the institu- tions participating in the registry and the clinical research coordinators (Takemoto N, Haratani K, Sakata T, Kiguchi A, Matsushita M). We also thank our colleagues from Osaka University Center of Medical Data Science and Advanced Clinical Epidemiology Investigator's Research Project for their providing insight and expertise for our research.

\section{Disclosure}

Conflicts of interest: None declared.

\section{References}

1. Cabinet Office, Government of Japan. The present and future situation of aging. Available at: https://www8.cao.go.jp/kourei/w hitepaper/w-2019/zenbun/pdf/1s1s_01.pdf. Accessed April 19, 2020.

2. Cabinet Office, Government of Japan. The international trend of aging. Available from. Available at: https://www8.cao.go.jp/kour ei/whitepaper/w-2019/zenbun/pdf/1s1s_02.pdf. Accessed April $19,2020$.

3. Tsuchihashi M, Tsutsui H, Kodama K, Kasagi F, Takeshita A. Clinical characteristics and prognosis of hospitalized patients with congestive heart failure--a study in Fukuoka, Japan. Jpn Circ J 2000; 64: 953-9.

4. Schockmel M, Agrinier N, Jourdain P, et al. Socioeconomic factors and mortality in diastolic heart failure. Eur J Clin Invest 2014; 44: 372-83.

5. Lu MLR, Davila CD, Shah M, et al. Marital status and living condition as predictors of mortality and readmissions among African Americans with heart failure. Int J Cardiol 2016; 222: 
313-8.

6. Tsuchihashi M, Tsutsui H, Kodama K, et al. Medical and socioenvironmental predictors of hospital readmission in patients with congestive heart failure. Am Heart J 2001; 142: E7.

7. Verma AK, Schulte PJ, Bittner V, et al. Socioeconomic and partner status in chronic heart failure: relationship to exercise capacity, quality of life, and clinical outcomes. Am Heart J 2017; 183: 54-61.

8. Kitamura T, Sakata Y, Nakatani D, et al. Living alone and risk of cardiovascular events following discharge after acute myocardial infarction in Japan. J Cardiol 2013; 62: 257-62.

9. Case RB, Moss AJ, Case N, McDermott M, Eberly S. Living alone after myocardial infarction. Impact on prognosis. JAMA 1992; 267: 515-9.

10. Tamiya N, Noguchi H, Nishi A, et al. Population ageing and wellbeing: lessons from Japan's long-term care insurance policy. Lancet 2011; 378: 1183-92.

11. Weiner J, World Health. Organization (WHO). The role of informal support in long-term care. In: Brodsky J, Habib J, Hirschfeld M, eds. Available at: https://www.who.int/chp/knowledge/pu blications/policy_issues_ltc.pdf. Accessed April 19, 2020. Key Policy Issues in Long-Term Care Geneva, Switzerland: World Health Organization; 2003.

12. Takabayashi K, Ikuta A, Okazaki Y, et al. Clinical characteristics and social frailty of super-elderly patients with heart failure The Kitakawachi clinical background and outcome of Heart Failure Registry. Circ J 2016; 81: 69-76.

13. Takabayashi K, Kitaguchi S, Iwatsu K, et al. A decline in activities of daily living due to acute heart failure is an independent risk factor of hospitalization for heart failure and mortality. $\mathrm{J}$ Cardiol 2019; 73: 522-9.

14. Ho KK, Pinsky JL, Kannel WB, Levy D. The epidemiology of heart failure: the Framingham Study. J Am Coll Cardiol 1993; 22 Supplement A: 6A-13A.

15. Lichstein E, Sharma A. Changing endpoints for heart failure studies. J Am Coll Cardiol 2018; 71: 2653-5.

16. Howie-Esquivel J, Spicer JG. Association of partner status and disposition with rehospitalization in heart failure patients. Am J Crit Care 2012; 21: e65-73.

17. Roe-Prior P. Sociodemographic variables predicting poor postdischarge outcomes for hospitalized elders with heart failure. Medsurg Nurs 2007; 16: 317-21.

18. Damiani G, Salvatori E, Silvestrini G, et al. Influence of socioeconomic factors on hospital readmissions for heart failure and acute myocardial infarction in patients 65 years and older: evidence from a systematic review. Clin Interv Aging 2015; 10 : 237-45.

19. Rodríguez-Artalejo F, Guallar-Castillón P, Herrera MC, et al. Social network as a predictor of hospital readmission and mortality among older patients with heart failure. J Card Fail 2006; 12: 621-7.

20. Horwich TB, Kalantar-Zadeh K, MacLellan RW, Fonarow GC. Albumin levels predict survival in patients with systolic heart failure. Am Heart J 2008; 155: 883-9.

21. Al-Najjar Y, Clark AL. Predicting outcome in patients with left ventricular systolic chronic heart failure using a nutritional risk index. Am J Cardiol 2012; 109: 1315-20.

22. Davis MA, Murphy SP, Neuhaus JM, Gee L, Quiroga SS. Living arrangements affect dietary quality for U.S. adults aged 50 years and older: NHANES III 1988-1994. J Nutr 2000; 130: 2256-64

23. Davis MA, Randall E, Forthofer RN, Lee ES, Margen S. Living arrangements and dietary patterns of older adults in the United States. J Gerontol 1985; 40: 434-42.

24. Suzuki W, Ogura S, Izumida N. Burden of family care-givers and the rationing in the Long-Term Care Insurance benefits of Japan. Singapore Econ Rev 2008; 53: 121-44.

25. Shimizutani S, Noguchi H. What accounts for the onerous care burden at home in Japan? Evidence from household data. Econ Anal Economic and Social Research Institute 2005; 175: 1-28.

26. Inoue N. Living alone, an important risk factor for cardiovascular disease. J Cardiol 2013; 62: 263-4.

27. Davidson PM, Cockburn J, Newton PJ, et al. Can a heart failure-specific cardiac rehabilitation program decrease hospitalizations and improve outcomes in high-risk patients? Eur J Cardiovasc Prev Rehabil 2010; 17: 393-402.

28. Tsutsui H, Tsuchihashi-Makaya M, Kinugawa S, Goto D, Takeshita A, JCARE-CARD Investigators. Clinical characteristics and outcome of hospitalized patients with heart failure in Japan. Circ J 2006; 70: 1617-23.

29. Sato N, Kajimoto K, Keida T, et al. Clinical features and outcome in hospitalized heart failure in Japan (from the ATTEND Registry). Circ J 2013; 77: 944-51.

30. Tsutsui H. JCS; 2017. JHFS 2017. Guidelines for Diagnosis and Treatment of Acute and Chronic Heart Failure. Available at: htt p://www.j-circ.or.jp/guideline/pdf/JCS2017_tsutsui_h.pdf. Accessed July 4, 2020. 